POS PROCEEDINGS

\title{
Internal gas target experiments at the LHC
}

\author{
P. Di Nezza ${ }^{* a}$ V. Carassiti, ${ }^{b}$ G. Ciullo,,${ }^{b, c}$ P. Lenisa,,${ }^{b, c}$ L.L. Pappalardo, ${ }^{b, c}$ E. Steffens, ${ }^{d}$ \\ and A. Vasilyev ${ }^{e}$ \\ ${ }^{a}$ INFN Laboratori Nazionali di Frascati, Frascati (Rome), Italy \\ ${ }^{b}$ INFN Ferrara, Italy \\ ${ }^{c}$ Department of Physics, University of Ferrara, Italy \\ ${ }^{d}$ Physics Dept., FAU Erlangen-Nürnberg, Erlangen, Germany \\ e PNPI Gatchina, Petersburg, Russia \\ E-mail: Pasquale.DiNezza@lnf.infn.it, vito@fe.infn.it, \\ ciullo@fe.infn.it, lenisa@fe.infn.it, pappalardo@fe.infn.it, \\ Erhard.Steffensefau.de, Vasilyev_aa@pnpi.nrcki.ru
}

\begin{abstract}
The physics opportunities offered by a fixed-target program using the proton and lead-ion beams of the LHC is wide and exciting. The LHC beams can provide the most energetic fixed-target measurements by $p p, p A$, and $P b A$ collisions at $\sqrt{s_{N N}}=72-115 \mathrm{GeV}$ probing unexplored regions of the kinematical plane, including the high- $x$ region. In the case of an unpolarized target the physics rich ranges from QCD to astroparticle, while the polarized target option opens the ground to novel measurements of TMDs, thereby contributing with a complementary approach to the understanding of the dynamics of the quark-gluon interplay inside the nucleon up to the 3-dimensional description of the nucleons. Among the main LHC experiments LHCb is the most suitable to host a fixed gas target. A description of the ongoing fixed-target proposals aimed at having the first LHC unpolarized data in Run3 and polarized data in Run4 will be discussed.
\end{abstract}

23rd International Spin Physics Symposium - SPIN2018 -

10-14 September, 2018

Ferrara, Italy

${ }^{*}$ Speaker. 


\section{Introduction}

Collisions of LHC beams on fixed targets open new exiting fields of investigation allowing one to study production of particles carrying a large fraction of the target nucleon momentum at an energy scale up to $115 \mathrm{GeV}$, in the nucleon-nucleon center-of-mass frame, paving the way to innovative and fundamental measurements in regions of the kinematic plane essentially unexplored [1][2], Fig.1.

A fixed gas target has the advantage of providing a broad variety of collision systems, providing novel probes for the study of the nucleon and nuclear structure, and for measurements of great interest to cosmic rays physics and dark matter search. Its extension to the polarized gas target will offer a unique opportunity to probe polarized quark and gluon parton distributions, especially at high $x$ and intermediate $Q^{2}$, where experimental data are still largely missing. Then, beside standard collinear parton distribution functions (PDFs), it will make possible to study multidimensional polarized parton distributions, that depend also on parton transverse momentum (transversemomentum-dependent PDFs, or TMDs).
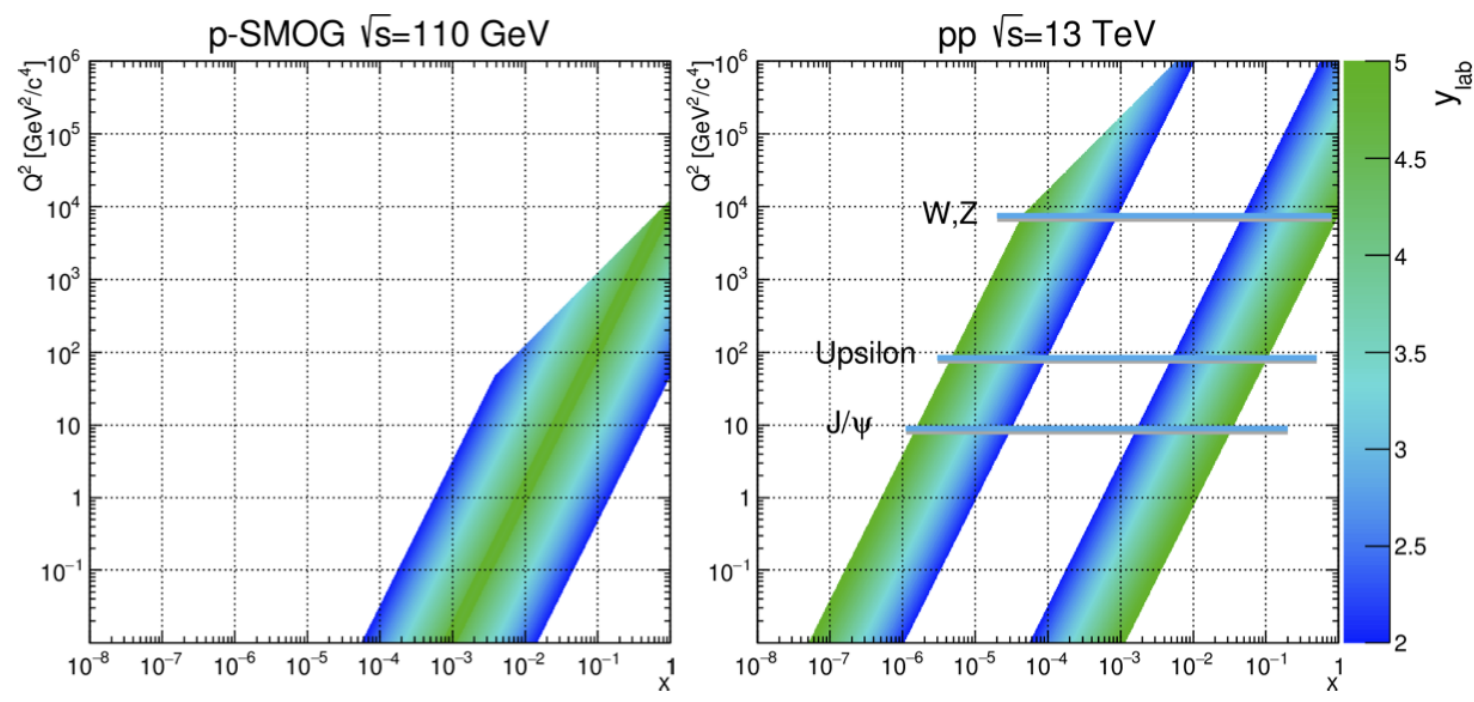

Figure 1: Accessible range in the $\left(x, Q^{2}\right)$ plane in (left) the fixed-target (SMOG) configuration, compared to (right) the collider configuration at $\mathrm{LHCb}[3]$.

\section{The physics case}

Among several advantages, we can identify the following main motivations for undertaking a fixed-target program at the LHC [3].

Relativistic heavy-ion collisions allow to investigate the high-density and high-temperature regime of QCD, where the production of heavy quarks is well suited to the study of the phase transition between ordinary hadronic matter and the Quark-Gluon Plasma (QGP). In fact, to investigate and disentangle all the physical processes contributing to nuclear collisions, measurements of heavy-quark production in a wide range of collision systems, collision energy, and kinematic 
acceptance are needed. In particular, the study of charmonium production in proton-induced reactions on various nuclear targets, where QGP is not expected to be formed, is needed to establish a robust baseline allowing for a correct interpretation of the QGP-induced suppression patterns observed in heavy-ion collisions.

The hydrodynamic description of the QCD medium, produced in heavy-ion collisions, can be tested in fixed-target data down to the energy of $\sqrt{s_{N N}} \sim 72 \mathrm{GeV}$ through measurements of flow observables and correlations for a variety of collision systems. Flow measurements will benefit from particle identification capabilities for pions, kaons and protons, as well as neutral particles $\phi, K_{S}^{0}$ and $\Lambda$. These studies can contribute in particular to shed light on the extension of the hydrodynamic description. A comprehensive fluid dynamical description of lower energy collisions with finite baryon density and an appropriate dynamical treatment will be important in the search for the critical point in the QCD phase diagram.

Understanding the bulk of particle production in high-energy hadronic collisions is an open challenge for the theory of strong interactions, since the contribution of non perturbative QCD effects in the soft or high-density regions becomes dominant, so that perturbative QCD can not be used to predict total inelastic cross sections. Beside the theoretical interest, knowledge of the multiplicity and spectra of particles produced in hadronic collisions, notably at large $x$, is important to model the underlying event for hadron-collider physics, and crucial to understand production of secondary particles in the propagation of cosmic rays through the interstellar gas (ISG) and the atmosphere. Available phenomenological models require tuning to experimental data, and the kinematic regime offered by LHC, in its fixed-target collisions, is unique. Moreover, the antimatter content in cosmic rays is a sensitive indirect probe for exotic sources of antimatter production, like dark matter annihilation. The AMS results for the antiproton over proton ratio [5] indicate a slight tension with predictions of the expected $\bar{p}$ fluxes due to secondary production in collisions between primary cosmic rays and the ISG, which is essentially composed by hydrogen and helium. In the $\bar{p}$ energy range 10 to $100 \mathrm{GeV}$, the largest uncertainty on such predictions is due to the limited knowledge of the $\bar{p}$ production cross-sections in the relevant processes. Since these cross-sections can be predicted in phenomenological models only within large uncertainties, computations of the expected $\bar{p}$ flux are based on extrapolations of existing measurements. The only available measurement comes from LHCb [6] showing the potentialities to substantially improve all the source of uncertainties in fixed target measurements.

The nucleon structure is traditionally parametrized in terms of PDFs, which, in their simplest (collinear) form, are functions of the longitudinal momentum fraction of quarks and gluons, expressed by the Bjorken- $x$ variable. Although tremendous advances have been made over the past decades in defining the quark and gluon dynamical substructure of the nucleon, the present knowledge of the PDFs still suffers from large uncertainties, especially at very-high and very-low $x$, leaving open fundamental questions about QCD and confinement. As an example, Fig. 2 shows the situation of the $u$ - and $d$-quark PDFs based on some of the latest parameterizations [7]. In many cases, the PDF uncertainties have become the limiting factor in the accuracy of the predictions for LHC measurements. This is particularly critical for the case of precision measurements of SM and BSM observables, where PDFs at high- $x$ constitute an important input for predictions. Among the various processes, Drell-Yan and weak-boson production are particularly suitable to reduce the uncertainties on the light quark and anti-quark PDFs in a wide kinematic region. The possibility 

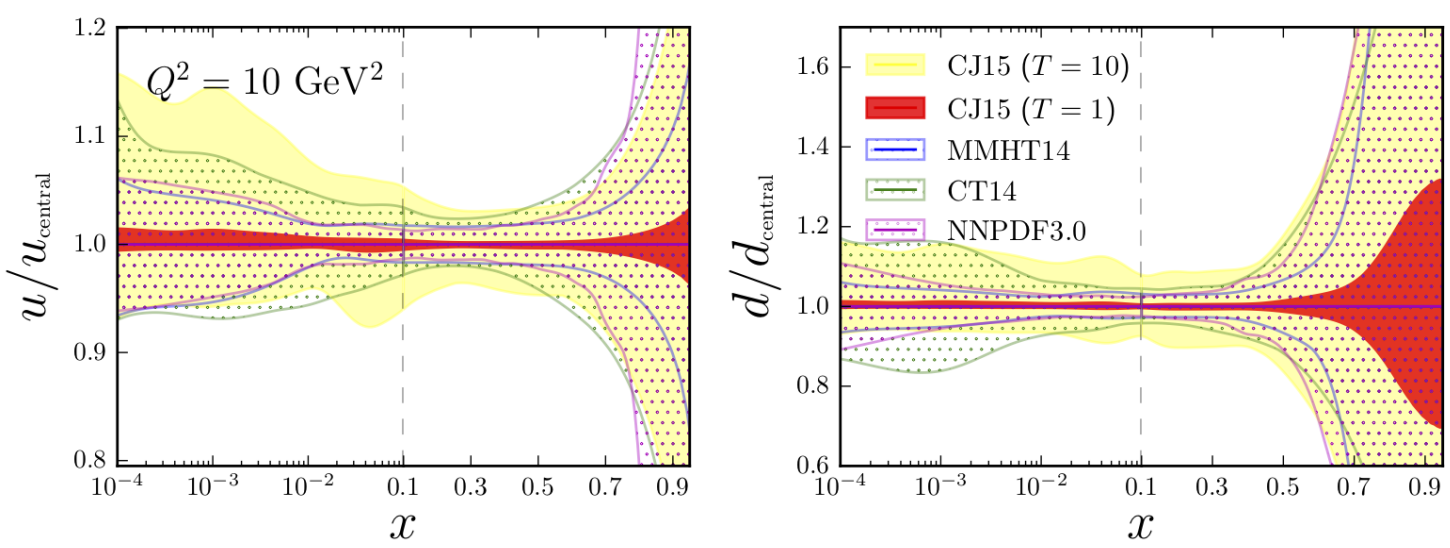

Figure 2: Ratio of $u$ (left) and $d$ (right) PDFs to the CJ15 parameterization central values for various PDF sets [7].

to study nuclear PDFs (nPDFs) in the high- $x$ region, where they are affected by relevant nuclear effects like the enhancement with respect to free nucleons around $x \sim 0.1$ known as antishadowing, and the EMC suppression at larger $x$ values, whose interpretation is still debated [4], is one of the key points of the proposed physics program. In particular, the PDFs for gluons and sea quarks, and the flavour-dependence of the nuclear effects are still poorly constrained. Moreover, measurements from fixed-target $p p$ collisions could be conclusive in establishing the amount of intrinsic charm content in the nucleons. The confirmation of this prediction from QCD would be of fundamental importance by itself, and would have consequences for present and future collider physics. The access to the gluon distributions is possible through the study of heavy-flavour production, which, in high-energy hadronic collisions, is dominantly generated by gluon-gluon interactions. In general, quarkonium production is extremely valuable to constrain gluon PDFs and nPDFs. Particularly effective probes are the $\mathrm{C}=+1$ quarkonia states. Among these, $\eta_{c}$ has never been studied in high-energy hadroproduction, and its production rate is expected to be substantial at the LHC. Of particular interest is the investigation of the gluon content of the neutron, as compared to the proton. This can be achieved e.g. by studying $J / \Psi$ or $\Upsilon$ production on both $\mathrm{H}_{2}$ and $\mathrm{D}_{2}$ targets.

Most of our knowledge about the collinear PDFs comes from decades of inclusive DIS experiments. Considering also the explicit dependence of PDFs on the parton transverse momenta, radically new perspectives in the exploration of the structure of the nucleon can be opened. Differently from the collinear PDFs, which only provide a 1-dimensional description of the nucleon structure in terms the longitudinal momentum fraction of partons, TMDs are sensitive to spin-orbit correlations inside the nucleon. They are thus indirectly sensitive to the still unknown parton orbital angular momentum, the main missing piece in the proton spin puzzle. In addition, they provide the possibility to map the parton densities in the 3-dimensional momentum space, spanned by the longitudinal momentum fraction $x$ and by the two transverse momentum components, allowing for a nucleon tomography in momentum space, Fig. 3. More generally, the knowledge of TMDs will lead to a significantly more profound and fundamental understanding of the complex dynamics of quarks and gluons in the non-perturbative regime of QCD. The study of the multidimensional par- 
tonic structure of the nucleon, including in particular polarization effects, can test our knowledge of QCD at an unprecedented level of sophistication, both in the perturbative and nonperturbative regime.

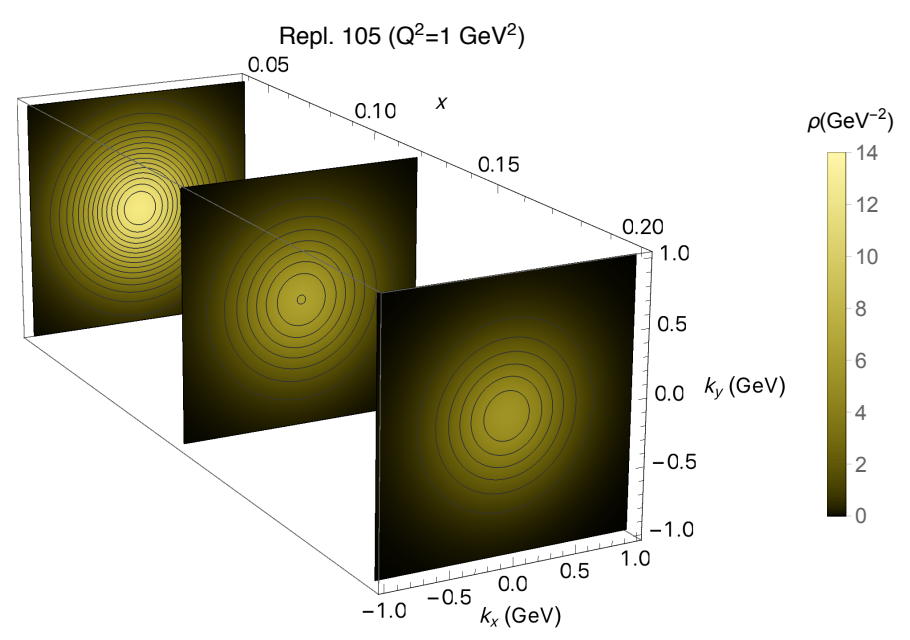

Figure 3: 3D rappresentation of the u-quark densities in momentum space [9].

It is also conceivable to go beyond the 3D description provided by TMDs and consider even higher-dimensional partonic distributions, corresponding to quantum phase-space distributions in mixed coordinate and momentum space. These Wigner distributions are extremely difficult to access directly, but very interesting ideas to solve this issue have been put forward recently, including the study of observables in $p p$ and $p A$ scattering processes [10,11].

For polarized nucleon targets, the transversity PDF is the most elusive of all quark PDFs. It describes the distribution of transversely polarized quarks in a transversely polarized proton target. It was introduced about 40 years ago and yet state-of-the-art extractions are limited to valence quark combinations and are performed at leading order only [12]. Recently, transversity received increasing attention because its integral, the so-called tensor charge, is needed to constrain beyondSM tensor couplings [13]. The tensor charge is considered to be one of the quantities best predicted by lattice QCD, but present extractions hint at a significant disagreement with expectations. At the LHC, transversity could be studied using transversely polarized hydrogen or deuterium targets. Several different channels are independently sensitive to the transversity PDF: dihadron production, hadron-in-jet production, (single polarized) Drell-Yan, transverse hyperon production.

Considering now TMDs, their study requires a profoundly different framework compared to standard collinear PDFs. Factorization theorems, universality and evolution equations are the cornerstones of our description of hadrons in terms of partonic distributions: they have been widely applied and tested for collinear PDFs, but not for TMDs. Stringent tests of the formalism require the comparison between different processes (lepton-hadron and hadron-hadron collisions) across a wide kinematic range.

In comparison with quark TMDs, the present knowledge of gluon TMDs is at an even lower stage. Although the theory framework is well consolidated, the experimental access is still extremely limited. Three gluon TMDs play a relevant role for a detailed comprehension of the 
internal structure of the nucleons: the unpolarized one (mapping the distribution of unpolarized gluons inside an unpolarized proton), the linearly polarized gluon TMD (mapping the distribution of gluons with a well-defined linear polarization inside an unpolarized proton), and the so-called gluon Sivers function (mapping the distribution of unpolarized gluons inside a transversely polarized proton). The first two TMDs will be accessible at LHC through the unpolarized fixed target data, at $\sqrt{s}=115 \mathrm{GeV}$. The gluon Sivers function (GSF) can be studied only with the polarized target. In particular, quarkonium production in fixed-target $p p$ interactions turns out to be an ideal observable to study the gluon TMDs. Among polarized TMDs, the Sivers function is particularly interesting. It gives rise to transverse single-spin asymmetries (SSAs) in semi-inclusive DIS as well as in Drell-Yan processes and offers the possibility to perform a stringent test of TMD factorization.

Transverse momentum spectra of the detected particles are the observables which can be used to extract the unpolarized gluon TMD, while azimuthal and Single-Spin Asymmetries can give access to the TMD of linearly polarized gluons and the GSF. The results with unpolarized target will be providing useful information on observables crucial for the extraction of unpolarized and linearly polarized TMDs, and at the same time will set the fundamentals to the measurements with polarized data.

Among the most important, and still open, theoretical issues, the QCD evolution of the gluon TMDs as well as the universality properties of the GSF are currently under active investigation.

Since transverse-momentum-dependent QCD factorization requires $p_{T}(Q) \ll M_{Q}$, where $Q$ denotes a heavy quark, the safest processes to be studied with a polarized hydrogen target are backto-back production of quarkonia and isolated photons or associated quarkonium production, where only the relative $p_{T}$ has to be small compared to $M_{Q}$.

\section{Unpolarized Target}

Among the main LHC experiments, LHCb is the only detector that can already run both in collider and fixed-target mode. The LHCb fixed-target system, called SMOG (System for Measuring the Overlap with Gas), was originally conceived for precise colliding-beams luminosity calibration [18]. The SMOG system allows to inject a low flow rate of noble gas into the vacuum vessel of the LHCb VErtex LOcator (VELO) detector. A temporary local pressure bump of about $10^{-7}$ mbar is obtained in the LHCb beam-pipe section (over a length of about $40 \mathrm{~m}$ around the nominal interaction point), which is about two order of magnitude higher than the nominal LHC vacuum pressure and one order of magnitude lower than the LHC vacuum interlock limit. The resulting beam-gas collision rate allows for a precise determination of the beam density profiles. As an additional important feature, SMOG gives the unique opportunity to operate $\mathrm{LHCb}$ in fixed target mode, and to study proton-nucleus and nucleus-nucleus collisions on various target types and at different centerof-mass energies. Several dedicated runs have already been performed since 2015 using He, Ar, or $\mathrm{Ne}$ targets with proton and $\mathrm{Pb}$ beams.

The SMOG2 project [14] constitutes an upgrade of the actual SMOG system. The core idea of SMOG2 is the use of a storage cell for the injected gas, to be installed at the upstream edge of the VELO, coaxial with the LHC beam. The proposed plan is to install the SMOG2 setup during the LHC Long Shutdown 2 (LS2) 2019-2020. The main advantage of SMOG2 is the possibility to reach effective areal densities (and thus luminosities) higher by a factor of about $8\left(\mathrm{H}_{2}\right)$ to 35 
(Ar) with respect to SMOG by injecting the same flow rate. A further increase of densities could be considered if compatible with the spectrometer occupancy.

A detailed physics program with a fixed target at LHCb is presented in a dedicated report of the Physics Beyond Collinders study group, Ref. [3]. The SMOG2 project presents various challenges, that are addressed in this document. In particular, the storage cell has to fulfil different requirements:

- it must fit into the limited space available inside the existing VELO vessel, upstream of the VELO detector;

- it must be openable (consisting of two halves), such to rigidly follow the motion of the two halves of the VELO detector (open position during beam injection and tuning, closed position during normal lumi operation);

- it must be light and thin, in order to keep the impact of the material budget in the proximity of the beam at a negligible level;

- it must ensure sufficient gas tightness along the sides in closed position, when the gas is flowing;

- it must have an openable system of Wake Field Suppressors (WFS) to ensure electrical continuity along the beam pipe and suppression of the wake fields;

- it must be properly coated to avoid instabilities by the formation of Electron Clouds.

The use of a tubular storage cell coaxial with the beam is the optimal choice for a gas target since, given a certain gas input, it allows to maximize the areal density seen by the beam.

Views of the storage cell arrangement inside the VELO vessel are presented in Fig. 4. The cell has a length of $200 \mathrm{~mm}$, a diameter of $10 \mathrm{~mm}$ (in closed position) and a thickness of $0.20 \mathrm{~mm}$. The gas is injected in the cell center by means of a small capillary.

The cell system is composed by a cylindrical WFS, a conical shape that allows for a smooth transition from the larger diameter of the upstream beam pipe to the smaller diameter of the cell tube $(10 \mathrm{~mm})$, an open-ended tube with wings and a contact piece of the WFS system which connects to the VELO detector box. The flexible parts are made by $\mathrm{CuBe}$, while the rigid parts are entirely made of $99.5 \%$ pure Aluminum.

For SMOG2 a Gas Feed System (GFS) with multiple injection lines and precise determination of the gas flow has been designed. The amount of gas injected can be accurately measured in order to precisely compute the target densities from the cell geometry and temperature. Beyond the constraints requested by LHC and LHCb, the GFS is a well established system, operated by the proponents in previous experiments [16][17].

Possible scenarios for the integrated luminosities to be collected with SMOG2 using different gas targets during the 3 years of Run3 data taking are reported in Ref. [3]. For sake of completeness we report here some of the integrated luminosities assumed for the yield estimates over the full Run3 period: $150 p b^{-1}$ for $p H_{2}, 45 p b^{-1}$ for $p A r, 22 p b^{-1}$ for $p X e$. Just as an example, considering only the $p A r$ collisions, we can collect the following yields: $J / \Psi \sim 15 \mathrm{M}, D^{0} \sim 150 \mathrm{M}, \Psi(2 S) \sim 150$ $\mathrm{k}, \Upsilon(1 S) \sim 7 \mathrm{k}$. 


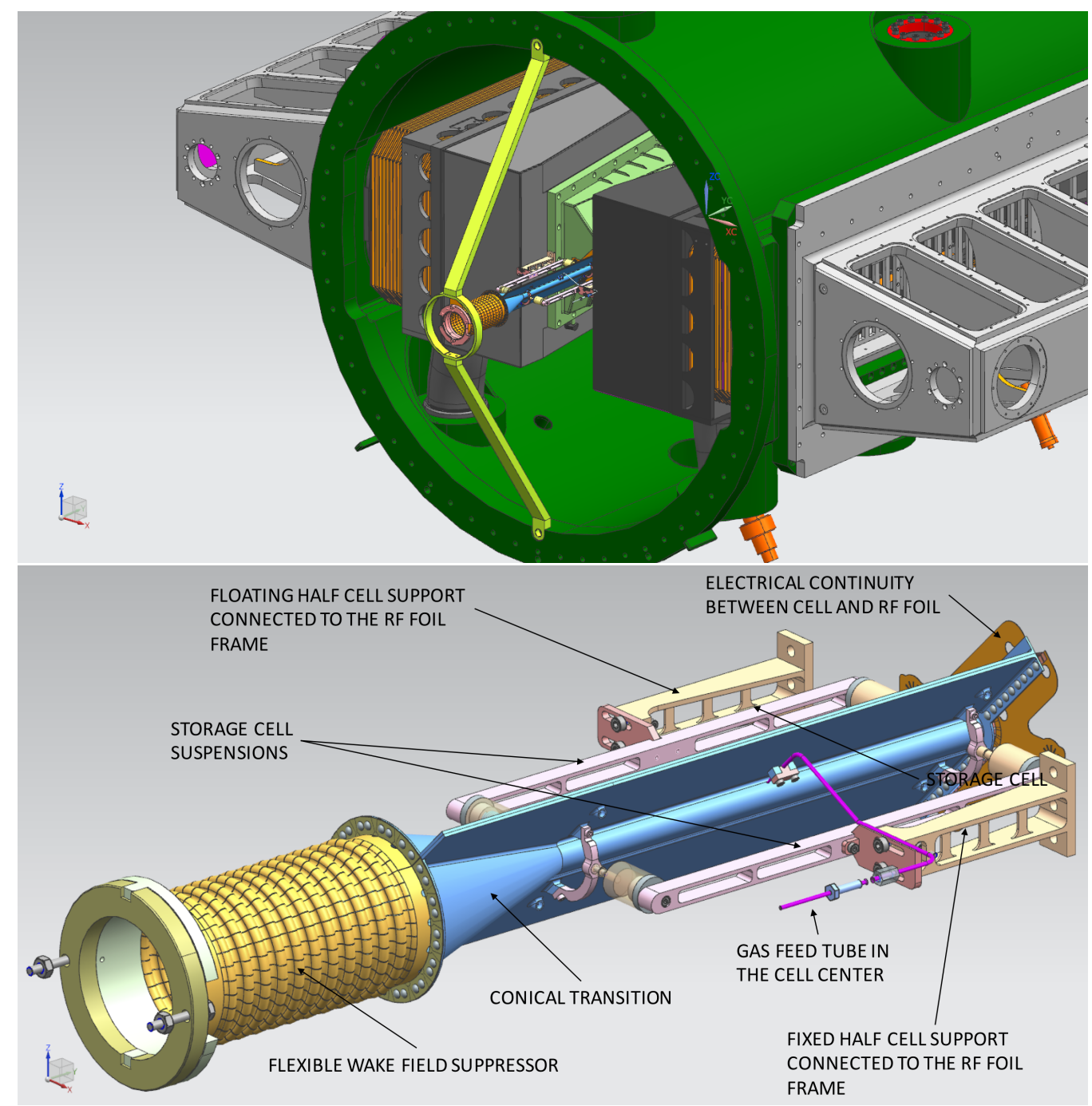

Figure 4: Top: SMOG2 storage cell mounted inside LHCb, in front of the VELO detector. Bottom: details of the storage cell.

When the target gas is injected, an additional beam-loss mechanism occurs, due to the beamgas collisions. The impact on the beam lifetime can be described in terms of the total beam-gas cross section and the expected luminosity. In all practical cases, the resulting beam partial lifetime largely exceeds the typical duration of a fill. For instance, one obtains about 97 days for $p A r$, and 22 days for $\mathrm{PbAr}$, while for a $\mathrm{pH}_{2}$ target the lifetime reduction exceeds 2000 days.

The SMOG2 R\&D is basically completed and the project has been approved by the $\mathrm{LHCb}$ collaboration and all the relevant LHC Working Groups gave the green light to proceed in order to finish the installation of the system at the end of LHC LS2.

\section{Polarized Target}

The realization of part of the rich physics program described above requires the implementation of a polarized gaseous target at the LHC [15]. Thanks to its forward spectrometer configu- 
ration, the $\mathrm{LHCb}$ detector constitutes the optimal choice among the existing experiments at LHC. Furthermore, the experience gained with SMOG2 will be fundamental for this further decisive step, which is being developed by the LHCSpin project [8]. Here a new and more complex system is required, to be located upstream of the SMOG2 system. The concept of the apparatus is based on the polarized target system used at the HERMES experiment (DESY) [16]. The setup will consist of four main components: an Atomic Beam Source (ABS), a Target Chamber (TC) hosting a storage cell, a diagnostic system, and an additional tracking detector.

The ABS generates a beam of polarized atomic gas ( $\mathrm{H}$ or $\mathrm{D})$ that is injected into a storage cell, in order to maximize the target areal density. The cell is placed inside the target chamber, into the LHC primary vacuum. The diagnostic system, including a Breit-Rabi polarimeter and a Gas Target Analyzer (TGA), allows to monitor both the fraction of atomic gas into the cell and the degree of polarization. The target chamber also hosts a transverse magnet $(\sim 300 \mathrm{mT})$, needed to define and keep the transverse polarization of the target gas. The HERMES polarized target has been successfully operated over a decade, with very high performances [16]. However, due to the limited space available upstream of the LHCb spectrometer, a new, more compact system has to be designed and constructed.

The ABS consists of a dissociator with a cooled nozzle, a system of sextupole magnets (SternGerlach apparatus) focusing the wanted (hydrogen or deuterium) hyperfine states into the feed tube of the cell, and adiabatic RF-transitions for setting and switching the target polarization between states of opposite sign. To maximize the stability and the degree of dissociation, a small percentage of oxygen $(0.1 \%-0.3 \%)$ could be added to the $\mathrm{H}_{2}$ gas. The water produced in the discharge improves the fraction of atoms in the beam which is formed by a system of cold nozzle and skimmer. A powerful differential pumping system with a total nominal pumping speed of the order of 15000 $1 / \mathrm{s}$ ensures low gas flow into the LHC vacuum system. The minimum amount of water travelling with the atomic beam will form a thin ice layer on the target cell internal walls, to be kept at about $100 \mathrm{~K}$, which reduces recombination and depolarization of the target gas to a high degree. The normal LHC vacuum pressure at the interaction point $\left(\sim 10^{-9}\right.$ mbar $)$ can be recovered within few minutes once the gas injection into the storage cell is stopped.

The Target Chamber (TC) will host a T-shaped openable storage cell. As for SMOG2, the cell, is divided in two halves. Its length will be of the order of $30 \mathrm{~cm}$, with a diameter of $1.0 \mathrm{~cm}$. The cell temperature could be set to values between 50 and $300 \mathrm{~K}$, although a temperature not higher than $100 \mathrm{~K}$ would be desirable to allow for the formation of an ice layer on the cell walls.

A diagnostic system is needed for continuously analyzing small samples of gas drawn from the target cell. It must consist of a Breit-Rabi Polarimeter (BRP), measuring the relative population of the injected hyperfine states, and a Target Gas Analyzer (TGA), detecting the molecular fraction and thus the degree of dissociation inside the cell. From the measurements of these parameters, and with appropriate corrections, the target polarization, as seen by the beam, can be deduced.

The arrangement of the Polarized Gas Target (PGT) in the beam line upstream of the VELO is shown schematically in Fig.5. An intermediate configuration for LHC Run3 including a valve for sectoring the LHC vacuum is shown which allows for the installation of fixed-target experiments upstream of VELO without breaking the vacuum in the VELO vessel. The PGT cannot be located close to or even inside the VELO vessel because of the high gas flow of atomic and recombined hydrogen which requires differential pumping on a separate target chamber. 


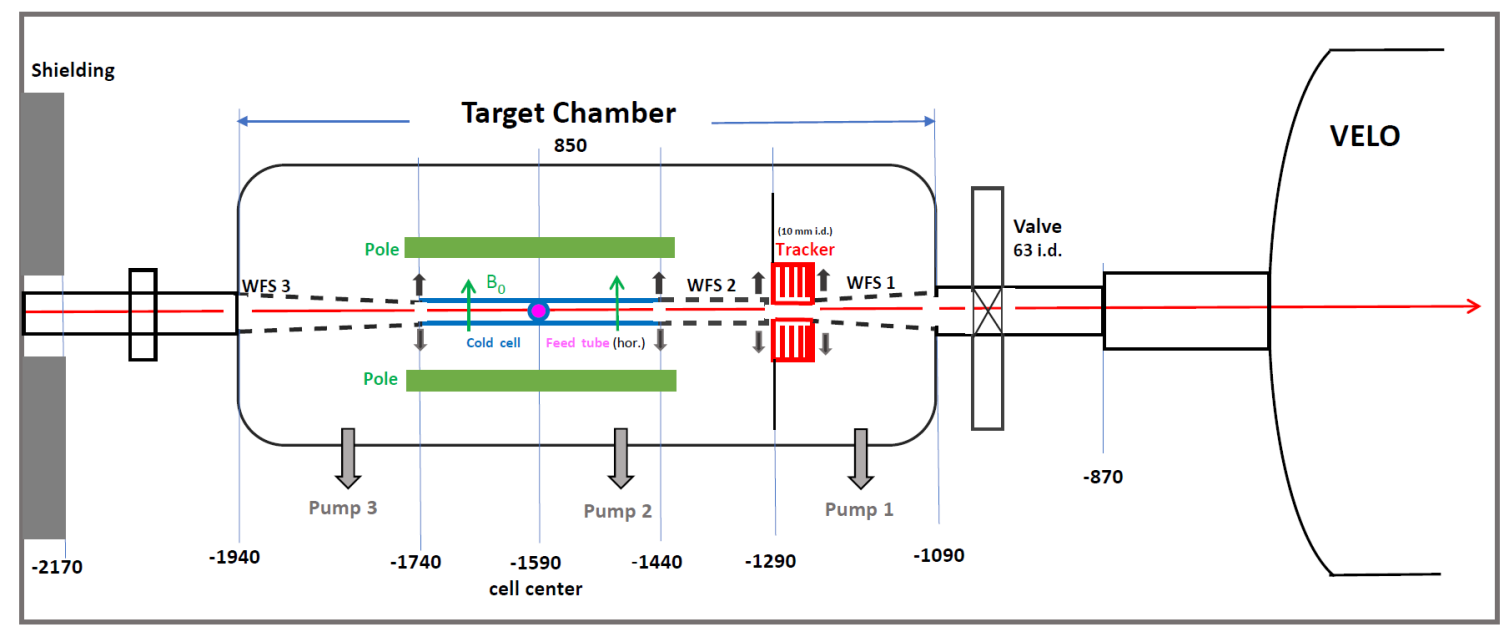

Figure 5: Schematic drawing showing the proposed PGT arrangement upstream of VELO with (right to left): new sectoring valve (installed in 2020), target chamber with conical WFS1 $(\mathrm{L}=20 \mathrm{~cm})$, Tracker (red) with $1 \mathrm{~cm}$ opening, gridded tube WFS2 $(\mathrm{L}=15 \mathrm{~cm})$ for differential pumping, cold cell $(\mathrm{L}=30 \mathrm{~cm})$ with beam tube and feed tube for injection of ABS beam in a vertical guide field $\mathrm{B}_{0}$ of about $0.3 \mathrm{~T}$. The different pumping stages are indicated. The openable components are denoted with black arrows. The nominal IP is within the VELO detector at $\mathrm{z}=0$.

Due to this distance between the cell and the VELO detector, an additional tracker has to be installed inside the TC, in order to supplement the tracking capabilities of the VELO in this upstream region. To maximize the acceptance, this new tracker must be located as close as possible to the beam. As a consequence, radiation hardness and high granularity are mandatory. Although the details of the detector have to be carefully studied with simulations, a preliminary concept has been elaborated, based on $3-5$ layers of Silicon pixel sensors.

Considering the geometry of the cell and assuming an ABS intensity of $6.5 \cdot 10^{16}$ atoms/s into the cell feed tube, corresponding to a recombined $H_{2}$ flow rate of about $2.5 \cdot 10^{-3} \mathrm{mbar} 1 / \mathrm{s}$ into the TC vacuum system, one obtains a central density $\rho_{0}=4.68 \cdot 10^{12} \mathrm{~cm}^{-3}$. For the case of polarized hydrogen, this results in an areal density of $\theta=1.21 \cdot 10^{14} \mathrm{H} \mathrm{cm}^{-2}$ at $T=100 \mathrm{~K}$, which is about two orders of magnitude higher than the density of the injected atomic beam. Assuming a (conservative) LHC proton beam intensity of $3.8 \cdot 10^{18} \mathrm{p} / \mathrm{s}$ for the LHC Run4, the resulting luminosity for $p H$ collisions is of the order of $L_{p H}=4.7 \cdot 10^{32} \mathrm{~cm}^{-2} s^{-1}$ at $100 \mathrm{~K}$.

An important parameter to be considered is the impact of the gas target on the beam lifetime $\tau_{p}$. Assuming a maximum value of $L_{p H}^{\max }=1.0 \cdot 10^{33} \mathrm{~cm}^{-2} s^{-1}$, the loss rate can be obtained by multiplying this instantaneous luminosity with the $p p$ total cross section $\sigma_{t o t}$ at a center of mass energy of $115 \mathrm{GeV}$, which can be estimated to be $0.05 \mathrm{~b}$. This corresponds to a minimal partial beam lifetime of $\tau_{p} \sim 77$ days, i.e. much longer than the duration of a typical fill. It is then possible to conclude that, as for the unpolarized gas target, also for the $\mathrm{pH}$ (and $\mathrm{pD}$ ) polarized case, additional beam losses caused by the target gas are completely negligible.

\section{Other proposals}

A fixed target program is being discussed also for the ALICE experiment [1][2]. However, due 
to cylindrical geometry of the spectrometer, and its reduced available space around the interaction region, different solutions than SMOG2 or LHCSpin are considered and the R\&D process is still in a developing phase.

\section{Conclusions}

Fixed target collisions at the LHC offer a unique opportunity for a laboratory for QCD and astroparticle in unexplored kinematic regions. $\mathrm{LHCb}$ is the most suitable experiment able to open this new field of research, also due to the previous experience with the SMOG system. The upgraded unpolarized gas target, SMOG2, is currently in the process of fabrication and validation. All the steps towards the final installation, scheduled for the LHC LS2, are carried out in close contact and collaboration with LHC experts from the various aspects involved (impedance, vacuum, machine protection, aperture).

The polarized target is the natural evolution of SMOG2. LHCSpin aims at installing a polarized gas target in front of the $\mathrm{LHCb}$ spectrometer, bringing for the first time polarization physics to the LHC. $\mathrm{LHCb}$ will then become the first experiment simultaneously running in collider and fixed-target mode with polarized targets, opening a whole new range of explorations to its exceptional spectrometer. The strong interest and support from the international theoretical community, together with the established experience of the experimental groups involved, will bring forward our knowledge of spin physics and QCD in a broad range of areas to an unprecedented level of sophistication.

\section{References}

[1] C. Hadjidakis at al., A Fixed-Target Programme at the LHC: Physics Case and Projected Performances for Heavy-Ion, Hadron, Spin and Astroparticle Studies, arXiv:1807.00603 [hep-ex].

[2] J.D. Bjorken et al., Community Support for A Fixed-Target Programme for the LHC, CERN-ESPP-Note, https://indico.cern.ch/event/765096/contributions/3295678/ .

[3] PBC Report, Physics opportunities with the fixed target program of the LHCb experiment using an unpolarized gas target, LHCb-PUB-2018-015.

[4] V. D. Burkert et al., The pressure distribution inside the proton, Nature vol. 557, 396-399, (2018).

[5] G. Giesen et al., AMS-02 antiprotons, at last! Secondary astrophysical component and immediate implications for Dark Matter, JCAP 09 (2015) 023, arXiv:1504.04276.

[6] LHCb collaboration, R. Aaij et al., Measurement of antiproton production in pHe collisions at $\sqrt{s_{N N}}$ $=110 \mathrm{GeV}$, arXiv:1808.06127, submitted to Phys. Rev. Lett.

[7] A. Accardi et al., Constraints on large-x parton distributions from new weak boson production and deep-inelastic scattering data, Phys. Rev. D93 (2016), no. 11, 114017, arXiv:1602.03154.

[8] C. A. Aidala et al., The LHCSpin project, CERN-ESPP-Note-2018-111, arXiv:1901.08002.

[9] A. Bacchetta et al., Extraction of partonic transverse momentum distributions from semi-inclusive deep-inelastic scattering, Drell-Yan and Z-boson production, JHEP 06 (2017) 081, [arXiv:1703.10157]. 
[10] Y. Hagiwara, Y. Hatta, B.-W. Xiao, and F. Yuan, Elliptic Flow in Small Systems due to Elliptic Gluon Distributions?, Phys. Lett. B771 (2017) 374-378, [arXiv:1701.04254].

[11] S. Bhattacharya, A. Metz, and J. Zhou, Generalized TMDs and the exclusive double Drell-Yan process, Phys. Lett. B771 (2017) 396-400, [arXiv:1702.04387].

[12] M. Radici and A. Bacchetta, First Extraction of Transversity from a Global Analysis of Electron-Proton and Proton-Proton Data, Phys. Rev. Lett. 120 (2018), no. 19 192001, [arXiv:1802.05212].

[13] A. Courtoy, S. Basseler, M. González-Alonso, and S. Liuti, Beyond-Standard-Model Tensor Interaction and Hadron Phenomenology, Phys. Rev. Lett. 115 (2015) 162001, [arXiv:1503.06814].

[14] V.Carassiti et al., SMOG2 Technical Proposal, CERN-PBC-Notes-2018-007, https://cds.cern.ch/record/2651269.

[15] E. Steffens, Design Considerations of a Polarized Gas Target for the LHC, Proceeding of Science (SPIN2018), https://pos.sissa.it/ (in print).

[16] HERMES Collaboration, A. Airapetian et. al., The HERMES polarized hydrogen and deuterium gas target in the HERA electron storage ring, Nucl. Instrum. Meth. A540 (2005) 68-101, [physics/0408137].

[17] V. Barone, et al., Antiproton-proton scattering experiments with polarization, hep-ex/0505054; http://collaborations.fz-juelich.de/ikp/pax/index.shtml;

[18] C. Barschel, Precision luminosity measurements at LHCb with beam-gas imaging, CERN-THESIS-2013-301, RWTH Aachen University, 2014. 